\title{
The Calculations of Topological Indices on Certain Networks
}

\author{
Jia-Bao Liu $\mathbb{D}$, ${ }^{1}$ Ting Zhang $\mathbb{D},{ }^{1}$ and Sakander Hayat $\mathbb{D}^{2}$ \\ ${ }^{1}$ School of Mathematics and Physics, Anhui Jianzhu University, Hefei 230601, China \\ ${ }^{2}$ Faculty of Engineering Sciences, GIK Institute of Engineering Sciences and Technology, Topi Swabi, \\ Khyber Pakhtunkhwa, Pakistan
}

Correspondence should be addressed to Jia-Bao Liu; liujiabaoad@163.com and Ting Zhang; zhangting1996@ahjzu.edu.cn

Received 28 November 2020; Revised 31 December 2020; Accepted 7 January 2021; Published 21 January 2021

Academic Editor: Shenggang Li

Copyright (c) 2021 Jia-Bao Liu et al. This is an open access article distributed under the Creative Commons Attribution License, which permits unrestricted use, distribution, and reproduction in any medium, provided the original work is properly cited.

\begin{abstract}
It is one of the core problems in the study of chemical graph theory to study the topological index of molecular graph and the internal relationship between its structural properties and some invariants. In recent years, topological index has been gradually applied to the models of QSAR and QSPR. In this work, using the definition of the ABC index, AZI index, GA index, the multiplicative version of ordinary first Zagreb index, the second multiplicative Zagreb index, and Zagreb index, we calculate the degree-based topological indices of some networks. Then, the above indices' formulas are obtained.
\end{abstract}

\section{Introduction}

The topological index is a numerical parameter in the structure graph of molecular compounds, and it can be used to predict the chemical and physical properties of molecules or to predict the biological activity [1-5]. In this paper, we mainly calculate several topological indices, which are invariants that can describe some properties of graph. The topological indices consist of three parts, namely, degreebased indices, spectrum-based indices, and distance-based indices; meanwhile, many indices based on both degree and distance are followed in $[6,7]$. The Kirchhoff index is based on the boiling point of kerosene, and other indices can predict the chemical and biological properties of some substances. We are dealing with some degree-based indices, such as, ABC index, AZI index, and GA index. We also calculate some indices for some chemical networks, examples include the first and the second Zagreb index and Zagreb index, by which we can predict the stability or others properties of some networks, such as $n$-dimensional silicate networks $\left(S_{n}\right)$, chain silicate networks $\left(C_{n}\right)$, hexagonal networks $\left(H_{n}\right), n$-dimensional honeycomb networks $\left(\mathrm{HC}_{n}\right)$, cellular networks $\left(\mathrm{CP}_{n}\right)$, and Sierpiński networks $(s(p, m))$. The graph of various networks are shown in Figures 1-6. In the rest of the paper, we made the following arrangements.
In Section 1, we introduce some indices and their backgrounds. In Section 2, we show the important results of this paper. In Section 3, we make a summary.

All graphs and networks are limited to simple undirected graphs. Let $G=(V(G), E(G))$ represent the vertex set and edge set of the networks, respectively. The degree of vertex $i$ is the number of edges associated with $i$, expressed by $d_{i}$. The standard notation and topological descriptors are mainly followed in [8].

According to the chemical molecules, the molecular graph is made up of atoms and bonds. The atom-bond connectivity index $\operatorname{ABC}(G)$ is written as

$$
\operatorname{ABC}(G)=\sum_{i j \in E(G)} \sqrt{\frac{d_{i}+d_{j}-2}{d_{i} \times d_{j}}},
$$

and posted by Estrada et al. [9]. The geometric-arithmetic index $\mathrm{GA}(G)$ is the valency-based topological index denoted by

$$
\mathrm{GA}(G)=\sum_{i j \in E(G)} \frac{2 \sqrt{d_{i} \times d_{j}}}{d_{i}+d_{j}},
$$

which is proposed by Furtula and Vukicević followed in [10]. Compared with other indices, geometric-arithmetic index is 


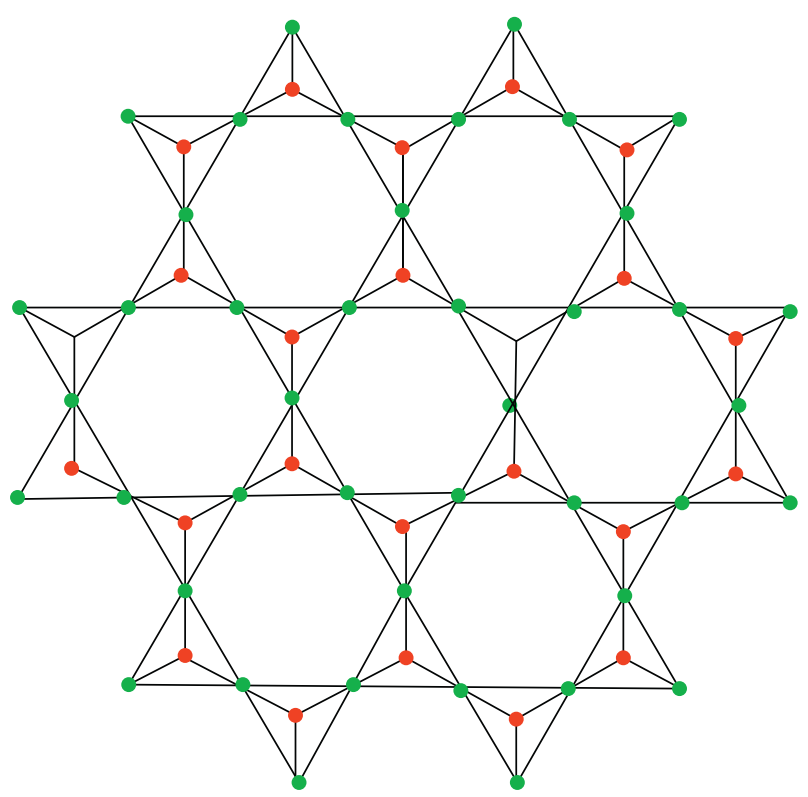

FIgURE 1: The silicate networks $S_{n}$.
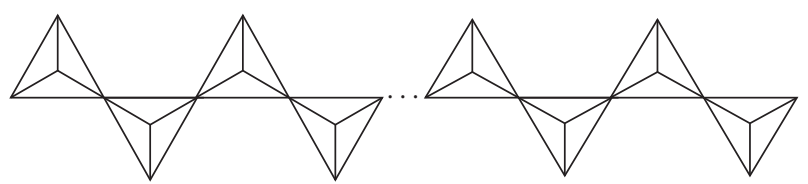

Figure 2: The chain silicate networks $C_{n}$.

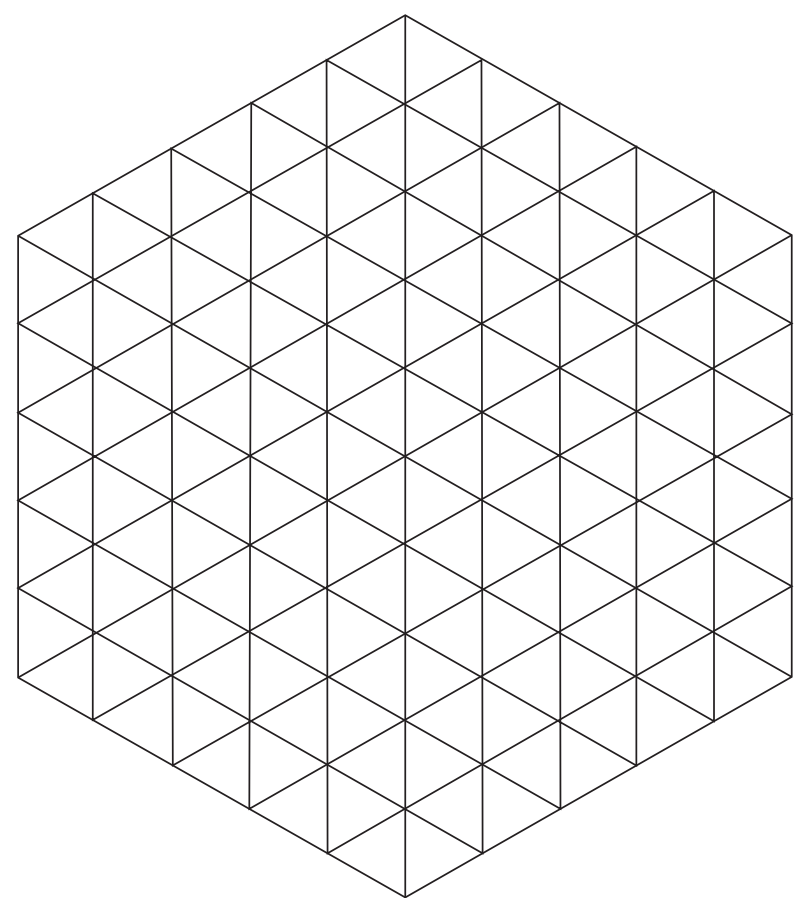

Figure 3: The hexagonal networks $H_{n}$.

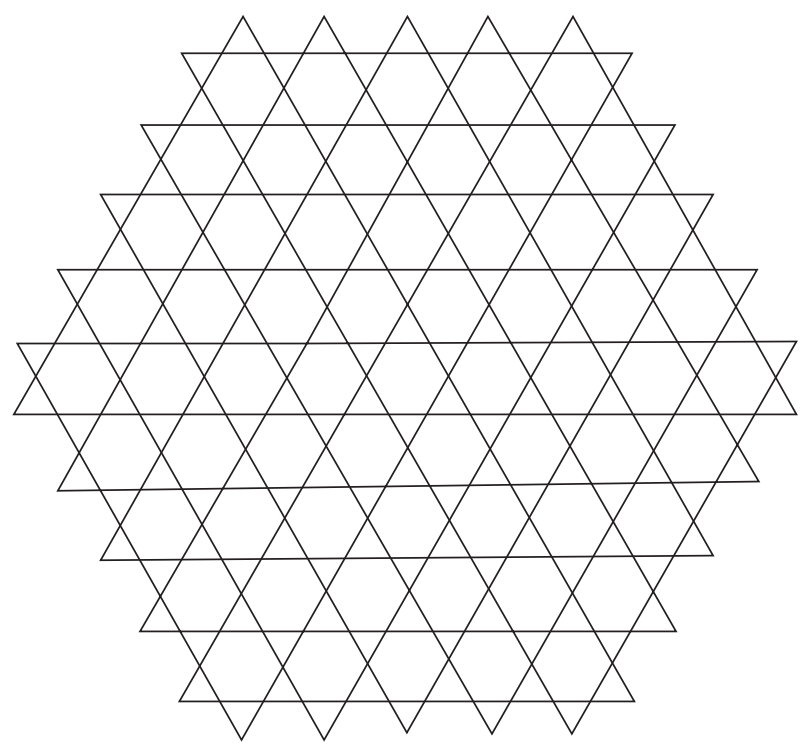

FIgURE 4: The oxide networks $O_{n}$.

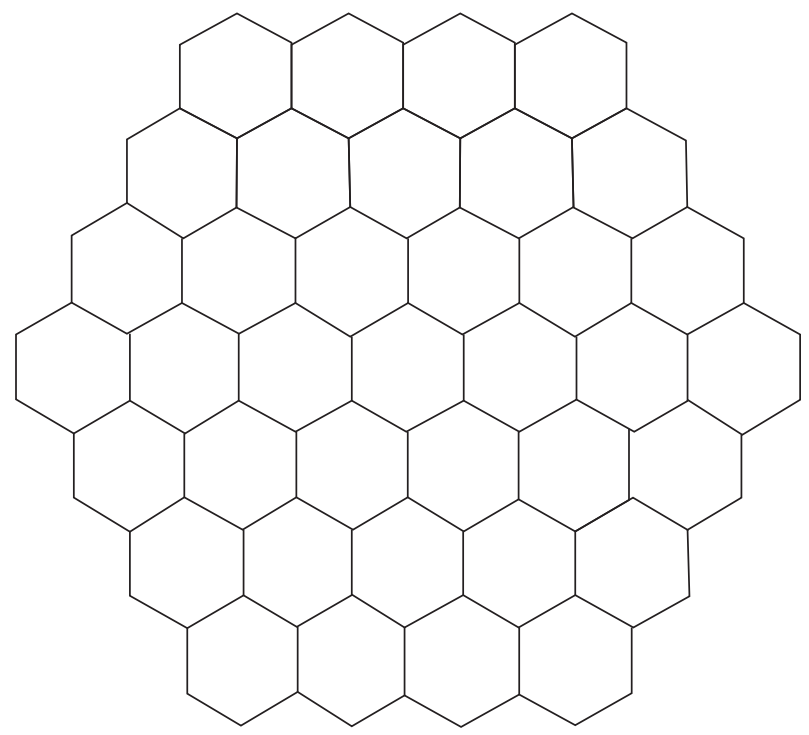

Figure 5: The cellular networks $\mathrm{CP}_{n}$.

better than other topological indices for predicting the physical and chemical properties of some substances, and more properties of $\mathrm{GA}(G)$ index is not introduced and readers can read the literature [11]. In 2010, Furtula et al. proposed AZI $(G)$ index [12], named as the augmented Zagred index, denoted by

$$
\operatorname{AZI}(G)=\sum_{i j \in E(G)}\left(\frac{d_{i} \times d_{j}}{d_{i}+d_{j}-2}\right)^{3} .
$$

The ordinary first Zagreb index of the multiplication version is represented as

$$
\Pi_{1}^{*}(G)=\prod_{i j \in E(G)}\left(d_{i}+d_{j}\right) .
$$




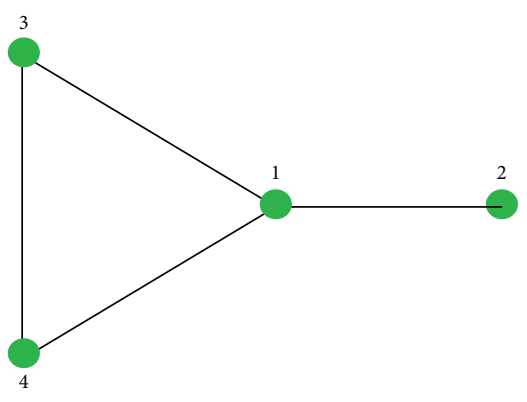

(a)

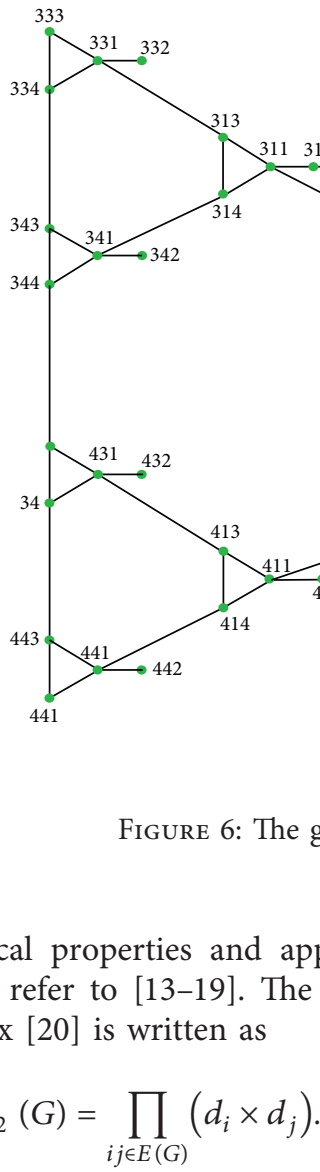

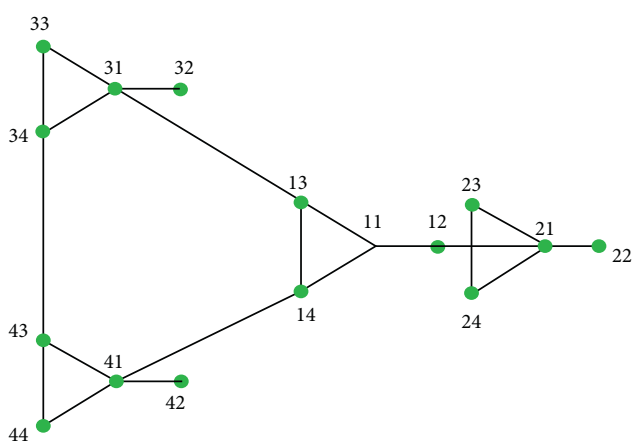

(b)
For the chemical properties and applications of this index, readers can refer to [13-19]. The second multiplication Zagreb index [20] is written as

$$
\Pi_{2}(G)=\prod_{i j \in E(G)}\left(d_{i} \times d_{j}\right) .
$$

On the basis of the Zagreb index, Azari et al. [21] put forward their general form and defined it as

$$
Z_{a, b}(G)=\sum_{i j \in E(G)}\left(d_{i}^{a} d_{j}^{b}+d_{i}^{b} d_{j}^{a}\right) .
$$

In 1972, the $F$ index was put forward, but there is little research on it. In 2015, B. Furtula and I. Gutman [22] redefined it as the forgotten topological index, or the $F$ index for short, and defined it as

$$
F(G)=\sum_{i j \varepsilon E(G)}\left(d_{i}^{2}+d_{j}^{2}\right) .
$$

About its related research, the reader may refer to [23-26].

\section{Main Results and Discussion}

In this section, according to the definition of the $A B C$ index, AZI index, GA index, multiplicative version of ordinary first Zagreb index, second multiplicative Zagreb index, and Zagreb index, we calculate the correlation index formula of several kinds of networks and get their concrete expressions.

Silicate is one of the most abundant minerals in the world. It is a mixture of metal compounds and sand [27]. $S_{n}$ represents a silicate network, where $n$ is the number of hexagons between the boundary and the center. Then, one has $\left|V\left(S_{n}\right)\right|=15 n^{2}+3 n,\left|\left(S_{n}\right)\right|=36 n^{2}$ and the following results.

According to the distribution of networks vertices, there are three sets of vertex division based on valencies, as $A_{1}, A_{2}$, and $A_{3}$. The set $A_{1}$ consists of $6 n$ edges $i j$, where $d_{i}=3$ and $d_{j}=3$. The set $A_{2}$ consists of $18 n^{2}+6 n$ edges $i j$, 
where $d_{i}=3$ and $d_{j}=6$. The set $A_{3}$ consists of $18 n^{2}-12 n$ edges $i j$, where $d_{i}=6$ and $d_{j}=6$.

Theorem 1. Suppose $G$ is a silicate network. Then,

$$
\begin{aligned}
\mathrm{GA}(G) & =(18+12 \sqrt{2}) n^{2}+(4 \sqrt{2}-6) n, \\
\mathrm{ABC}(G) & =(3 \sqrt{14}+3 \sqrt{10}) n^{2}+(4+\sqrt{14}-2 \sqrt{10}) n, \\
\operatorname{AZI}(G) & =\left(\frac{18^{4}}{7^{3}}+\frac{18^{4}}{5^{3}}\right) n^{2}+\left(\frac{3^{7}}{2^{5}}+\frac{6 \times 18^{3}}{7^{3}}-\frac{12 \times 18^{3}}{5^{3}}\right) n .
\end{aligned}
$$

Proof. Let $G$ be a silicate network. Then, one has

$$
\begin{aligned}
& \mathrm{GA}(G)=\sum_{i j \in E(G)} \frac{2 \sqrt{d_{i} \times d_{j}}}{d_{i}+d_{j}} \\
& =6 n \times \frac{2 \sqrt{3 \times 3}}{3+3}+\left(18 n^{2}+6 n\right) \times \frac{2 \sqrt{3 \times 6}}{3+6} \\
& +\left(18 n^{2}-12 n\right) \times \frac{2 \sqrt{6 \times 6}}{6+6} \\
& =6 n+\left(18 n^{2}+6 n\right) \times \frac{2 \sqrt{2}}{3}+\left(18 n^{2}-12 n\right) \\
& =(18+12 \sqrt{2}) n^{2}+(4 \sqrt{2}-6) n, \\
& \operatorname{ABC}(G)=\sum_{i j \in E(G)} \sqrt{\frac{d_{i}+d_{j}-2}{d_{i} \times d_{j}}} \\
& =6 n \times \sqrt{\frac{3+3-2}{3 \times 3}}+\left(18 n^{2}+6 n\right) \times \sqrt{\frac{3+6-2}{3 \times 6}}+\left(18 n^{2}-12 n\right) \times \sqrt{\frac{6+6-2}{6 \times 6}} \\
& =6 n \times \frac{2}{3}+\left(18 n^{2}+6 n\right) \times \frac{\sqrt{14}}{6}+\left(18 n^{2}-12 n\right) \times \frac{\sqrt{10}}{6} \\
& =(3 \sqrt{14}+3 \sqrt{10}) n^{2}+(4+\sqrt{14}-2 \sqrt{10}) n, \\
& \operatorname{AZI}(G)=\sum_{i j \in E(G)}\left(\frac{d_{i} \times d_{j}}{d_{i}+d_{j}-2}\right)^{3} \\
& =6 n \times\left(\frac{3 \times 3}{3+3-2}\right)^{3}+\left(18 n^{2}+6 n\right) \times\left(\frac{3 \times 6}{3+6-2}\right)^{3}+\left(18 n^{2}-12 n\right) \times\left(\frac{6 \times 6}{6+6-2}\right)^{3} \\
& =6 n \times\left(\frac{9}{4}\right)^{3}+\left(18 n^{2}+6 n\right) \times\left(\frac{18}{7}\right)^{3}+\left(18 n^{2}-6 n\right) \times\left(\frac{18}{5}\right)^{3} \\
& =\left(\frac{18^{4}}{7^{3}}+\frac{18^{4}}{5^{3}}\right) n^{2}+\left(\frac{3^{7}}{2^{5}}+\frac{6 \times 18^{3}}{7^{3}}-\frac{12 \times 18^{3}}{5^{3}}\right) n .
\end{aligned}
$$

Hexagonal networks is written as $H_{n}$, which is composed of $n$ hexagons. According to the relationship of degree series, we mainly calculate the following indices. One can refer to more research on hexagon networks [28-31].

Similarly, according to the degree distribution of the Hexagonal networks vertices, there are five sets of vertex division based on valencies, as $A_{1}, A_{2}, A_{3}, A_{4}$, and $A_{5}$. The set $A_{1}$ consists of 12 edges $i j$, where $d_{i}=3$ and $d_{j}=4$. The set $A_{2}$ consists of 6 edges $i j$, where $d_{i}=3 \mathrm{~s} d_{j}=6$. The set $A_{3}$ consists of $6 n-18$ edges $i j$, where $d_{i}=4$ and $d_{j}=4$. The set $A_{4}$ consists of $12 n-24$ edges $i j$, where $d_{i}=4$ and $d_{j}=6$. The set $A_{5}$ consists of $9 n^{2}-33 n+30$ edges $i j$, where $d_{i}=6$ and $d_{j}=6$. 
Theorem 2. Suppose $G$ is a hexagonal network. Then,

$$
\begin{aligned}
& \mathrm{GA}(G)=9 n^{2}+\left(\frac{24 \sqrt{6}}{5}-27\right) n+12+4 \sqrt{2}+\frac{48}{35}(5 \sqrt{3}-7 \sqrt{6}), \\
& \mathrm{ABC}(G)=\frac{3 \sqrt{10}}{2} n^{2}+\left(\frac{3}{2} \sqrt{6}+4 \sqrt{3}-\frac{11 \sqrt{10}}{2}\right) n+\left(2 \sqrt{15}+\sqrt{14}-\frac{9 \sqrt{6}}{2}-8 \sqrt{3}+5 \sqrt{10}\right), \\
& \mathrm{AZI}(G)=\frac{9 \times 18^{3}}{5^{3}} n^{2}+\left(\frac{2 \times 8^{3}}{9}+324-\frac{33 \times 18^{3}}{5^{3}}\right) n+\left(\frac{12^{4}}{5^{3}}+\frac{6 \times 18^{3}}{7^{3}}-\frac{2 \times 8^{3}}{3}+\frac{6 \times 18^{3}}{25}-648\right) .
\end{aligned}
$$

Proof. Only consider that $H_{n}$ is an $n$-dimensional hexagonal networks. So, $\left|V\left(H_{n}\right)\right|=3 n^{2}-3 n+1,\left|E\left(H_{n}\right)\right|=9 n^{2}-$ $15 n+6$. Thus,

$$
\begin{aligned}
& \mathrm{GA}(G)=\sum_{i j \in E(G)} \frac{2 \sqrt{d_{i} \times d_{j}}}{d_{i}+d_{j}} \\
& =12 \times \frac{2 \times \sqrt{3 \times 4}}{3+4}+6 \times \frac{2 \times \sqrt{3 \times 6}}{3+6}+(6 n-18) \times \frac{2 \times \sqrt{4 \times 4}}{4+4} \\
& +(12 n-24) \times \frac{2 \times \sqrt{4 \times 6}}{4+6}+\left(9 n^{2}-33 n+30\right) \times \frac{2 \times \sqrt{6 \times 6}}{6+6} \\
& =12 \times \frac{4 \sqrt{3}}{7}+6 \times \frac{2 \times 3 \sqrt{2}}{9}+(6 n-18) \times \frac{2 \times 4}{8}+(12 n-24) \\
& \times \frac{4 \sqrt{6}}{10}+\left(9 n^{2}-33 n+30\right) \times \frac{2 \times \sqrt{6 \times 6}}{6+6} \\
& =9 n^{2}+\left(\frac{24 \sqrt{6}}{5}-27\right) n+12+4 \sqrt{2}+\frac{48}{35}(5 \sqrt{3}-7 \sqrt{6}), \\
& \operatorname{ABC}(G)=\sum_{i j \in E(G)} \sqrt{\frac{d_{i}+d_{j}-2}{d_{i} \times d_{j}}} \\
& =12 \times \sqrt{\frac{3+4-2}{3 \times 4}}+6 \times \sqrt{\frac{3+6-2}{3 \times 6}}+(6 n-18) \times \sqrt{\frac{4+4-2}{4 \times 4}}+(12 n-24) \times \sqrt{\frac{4+6-2}{4 \times 6}} \\
& +\left(9 n^{2}-33 n+30\right) \times \sqrt{\frac{6+6-2}{6 \times 6}} \\
& =12 \times \sqrt{\frac{5}{12}}+6 \times \sqrt{\frac{7}{18}}+(6 n-18) \times \sqrt{\frac{6}{16}}+(12 n-24) \times \sqrt{\frac{8}{24}}+\left(9 n^{2}-33 n+30\right) \times \sqrt{\frac{10}{36}} \\
& =\frac{3 \sqrt{10}}{2} n^{2}+\left(\frac{3}{2} \sqrt{6}+4 \sqrt{3}-\frac{11 \sqrt{10}}{2}\right) n+\left(2 \sqrt{15}+\sqrt{14}-\frac{9 \sqrt{6}}{2}-8 \sqrt{3}+5 \sqrt{10}\right) \text {, }
\end{aligned}
$$




$$
\begin{aligned}
\operatorname{AZI}(G)= & \sum_{i j \in E(G)}\left(\frac{d_{i} \times d_{j}}{d_{i}+d_{j}-2}\right)^{3} \\
= & 12 \times\left(\frac{3 \times 4}{3+3-2}\right)^{3}+6 \times\left(\frac{3 \times 6}{3+6-2}\right)^{3}+(6 n-18) \times\left(\frac{4 \times 4}{4+4-2}\right)^{3} \\
& +(12 n-24) \times\left(\frac{4 \times 6}{4+6-2}\right)^{3}+\left(9 n^{2}-33 n+30\right) \times\left(\frac{6 \times 6}{6+6-2}\right)^{3} \\
= & 12 \times \frac{12^{3}}{5^{3}}+6 \times \frac{18^{3}}{7^{3}}+(6 n-18) \times \frac{16^{3}}{6^{3}}+(12 n-24) \times \frac{24^{3}}{8^{3}}+\left(9 n^{2}-33 n+30\right) \times \frac{36^{3}}{10^{3}} \\
= & \frac{9 \times 18^{3}}{5^{3}} n^{2}+\left(\frac{2 \times 8^{3}}{9}+324-\frac{33 \times 18^{3}}{5^{3}}\right) n+\left(\frac{12^{4}}{5^{3}}+\frac{6 \times 18^{3}}{7^{3}}-\frac{2 \times 8^{3}}{3}+\frac{6 \times 18^{3}}{25}-648\right) .
\end{aligned}
$$

At present, we discuss about another member of the silicate networks and the chain silicate networks, which is a linear combination of $n$ tetrahedrons, referred to as $C_{n}$. In the same way, the edges of the silicate networks can be divided into three sets of vertex division based on valencies, as $A_{1}, A_{2}$, and $A_{3}$. For $n=1$, the set $A_{1}$ consists of 6 edges $i j$, where $d_{i}=3$ and $d_{j}=3$, the set $A_{2}$ consists of 0 edges $i j$, where $d_{i}=3$ and $d_{j}=6$, and the set $A_{3}$ consists of 0 edges $i j$, where $d_{i}=6$ and $d_{j}=6$. For $n \geq 2$, the set $A_{1}$ consists of $n+4$ edges $i j$, where $d_{i}=3$ and $d_{j}=3$, the set $A_{2}$ consists of $4 n-$ 2 edges $i j$, where $d_{i}=3$ and $d_{j}=6$, and the set $A_{3}$ consists of $n-2$ edges $i j$, where $d_{i}=6$ and $d_{j}=6$.

Theorem 3. Suppose $G$ is a chain silicate network with $6 n$ edges and $3 n+1$ vertices. Then,

$$
\begin{aligned}
& \mathrm{GA}(G)= \begin{cases}6, & n=1, \\
\left(2+\frac{8 \sqrt{2}}{3}\right) n+\left(2-\frac{4 \sqrt{2}}{3}\right), & n \geq 2,\end{cases} \\
& \operatorname{ABC}(G)= \begin{cases}4, & n=1, \\
\left(\frac{4+4 \sqrt{14}+\sqrt{10}}{6}\right) n+\frac{8-\sqrt{14}-\sqrt{10}}{3}, & n \geq 2,\end{cases} \\
& \operatorname{AZI}(G)= \begin{cases}\frac{3^{7}}{2^{5}}, & n=1, \\
\left(\frac{9^{3}}{4^{3}}+\frac{4 \times 18^{3}}{7^{3}}+\frac{18^{3}}{5^{3}}\right) n+\left(\frac{9^{3}}{16}-\frac{2 \times 18^{3}}{7^{3}}-\frac{2 \times 18^{3}}{5^{3}}\right), & n \geq 2 .\end{cases}
\end{aligned}
$$


Proof. Let $G$ be a chain silicate network. Then,

$$
\mathrm{GA}(G)=\sum_{i j \in E(G)} \frac{2 \sqrt{d_{i} \times d_{j}}}{d_{i}+d_{j}},
$$

where

$$
\begin{aligned}
n=1, \quad \mathrm{GA}(G)= & 6 \times \frac{2 \sqrt{3 \times 3}}{3+3}=6, \\
n \geq 2, \quad \mathrm{GA}(G)= & (n+4) \times \frac{2 \sqrt{3 \times 3}}{3+3} \\
& +(4 n-2) \times \frac{2 \sqrt{3 \times 6}}{3+6}+(n-2) \times \frac{2 \sqrt{6 \times 6}}{6+6} \\
= & (n+4)+(4 n-2) \times \frac{2 \sqrt{2}}{3}+(n-2) \\
= & \left(2+\frac{8 \sqrt{2}}{3}\right) n+\left(2-\frac{4 \sqrt{2}}{3}\right), \\
\operatorname{ABC}(G)= & \sum_{i j \in E(G)} \sqrt{\frac{d_{i}+d_{j}-2}{d_{i} \times d_{j}}},
\end{aligned}
$$

where

$$
\begin{aligned}
n=1, \quad \operatorname{ABC}(G)= & 6 \times \sqrt{\frac{3+3-2}{3 \times 3}=4,} \\
n \geq 2, \quad \operatorname{ABC}(G)= & (n+4) \times \sqrt{\frac{3+3-2}{3 \times 3}} \\
& +(4 n-2) \times \sqrt{\frac{3+6-2}{3 \times 6}}+(n-2) \\
& \times \sqrt{\frac{6+6-2}{6 \times 6}} \\
= & \frac{2}{3}(n+4)+(4 n-2) \times \frac{\sqrt{14}}{6} \\
& +(n-2) \times \frac{\sqrt{10}}{6} \\
= & \left(\frac{4+4 \sqrt{14}+\sqrt{10}}{6}\right) n+\frac{8-\sqrt{14}-\sqrt{10}}{3}, \\
\operatorname{AZI}(G)= & \sum_{i j \in E(G)}\left(\frac{d_{i} \times d_{j}}{d_{i}+d_{j}-2}\right)^{3},
\end{aligned}
$$

where

$$
\begin{aligned}
n=1, \quad \operatorname{AZI}(G)= & 6 \times\left(\frac{3 \times 3}{3+3-2}\right)^{3}=\frac{3^{7}}{2^{5}} \\
n \geq 2, \quad \operatorname{AZI}(G)= & (n+4) \times\left(\frac{3 \times 3}{3+3-2}\right)^{3}+(4 n-2) \\
& \times\left(\frac{3 \times 6}{3+6-2}\right)^{3}+(n-2) \times\left(\frac{6 \times 6}{6+6-2}\right)^{3} \\
= & (n+4) \frac{9^{3}}{4^{3}}+(4 n-2) \times \frac{18^{3}}{7^{3}} \\
& +(n-2) \times \frac{36^{3}}{10^{3}} \\
= & \left(\frac{9^{3}}{4^{3}}+\frac{4 \times 18^{3}}{7^{3}}+\frac{18^{3}}{5^{3}}\right) n \\
& +\left(\frac{9^{3}}{16}-\frac{2 \times 18^{3}}{7^{3}}-\frac{2 \times 18^{3}}{5^{3}}\right) .
\end{aligned}
$$

Oxide networks play an important role in silicate networks. When the silicon atoms in the silicate networks are removed, the oxide networks are obtained. The $n$-dimensional oxide networks are defined as $O_{n}$. By observing the edge division of oxide networks, there are two sets of vertex division based on valencies, as $A_{1}$ and $A_{2}$. The set $A_{1}$ consists of $12 n$ edges $i j$, where $d_{i}=2$ and $d_{j}=4$. The set $A_{2}$ consists of $18 n^{2}-12 n$ edges $i j$, where $d_{i}=4$ and $d_{j}=4$.

Theorem 4. Suppose $G$ is an oxide network with $18 n^{2}$ edges and $9 n^{2}+3 n$ vertices. Then,

$$
\begin{aligned}
\mathrm{GA}(G) & =18 n^{2}+(8 \sqrt{2}-12) n, \\
\operatorname{ABC}(G) & =\frac{9 \sqrt{6}}{2} n^{2}+(6 \sqrt{2}-3 \sqrt{6}) n, \\
\operatorname{AZI}(G) & =\frac{1024}{3} n^{2}-\frac{1184}{9} n .
\end{aligned}
$$

Proof. Let $G$ be an oxide network. Then, one has 


$$
\begin{aligned}
& \mathrm{GA}(G)=\sum_{i j \in E(G)} \frac{2 \sqrt{d_{i} \times d_{j}}}{d_{i}+d_{j}} \\
& =12 n \times \frac{2 \sqrt{2 \times 4}}{2+4}+\left(18 n^{2}-12 n\right) \times \frac{2 \sqrt{4 \times 4}}{4+4} \\
& =18 n^{2}+(8 \sqrt{2}-12) n, \\
& \operatorname{ABC}(G)=\sum_{i j \in E(G)} \sqrt{\frac{d_{i}+d_{j}-2}{d_{i} \times d_{j}}} \\
& =12 n \times \sqrt{\frac{2+4-2}{2 \times 4}}+\left(18 n^{2}-12 n\right) \times \sqrt{\frac{4+4-2}{4 \times 4}} \\
& =12 n \times \frac{\sqrt{2}}{2}+\left(18 n^{2}-12 n\right) \times \frac{\sqrt{6}}{4} \\
& =\frac{9 \sqrt{6}}{2} n^{2}+(6 \sqrt{2}-3 \sqrt{6}) n, \\
& \operatorname{AZI}(G)=\sum_{i j \in E(G)}\left(\frac{d_{i} \times d_{j}}{d_{i}+d_{j}-2}\right)^{3} \\
& =12 n \times\left(\frac{2 \times 4}{2+4-2}\right)^{3}+\left(18 n^{2}-12 n\right) \times\left(\frac{4 \times 4}{4+4-2}\right)^{3} \\
& =12 n \times 2^{3}+\left(18 n^{2}-12 n\right) \times \frac{8^{3}}{27} \\
& =\frac{1024}{3} n^{2}-\frac{1184}{9} n
\end{aligned}
$$

Cellular networks is mainly composed of three parts: mobile station, network subsystem, and base station subsystem, denoted by $\mathrm{CP}_{n}$. It plays an important role in computer graphics and in chemistry. Meanwhile, it also can be characterized as benzene hydrocarbons. In the same way, the edges of cellular networks can be divided into three sets based on valencies, as $A_{1}, A_{2}$, and $A_{3}$. The set $A_{1}$ consists of 6 edges $i j$, where $d_{i}=2$ and $d_{j}=2$. The set $A_{2}$ consists of $12 n-12$ edges $i j$, where $d_{i}=2$ and $d_{j}=3$. The set $A_{3}$ consists of $9 n^{2}-15 n+6$ edges $i j$, where $d_{i}=3$ and $d_{j}=3$.

Theorem 5. Suppose $G$ is a cellular network with $6 n^{2}$ edges and $9 n^{2}-3 n$ vertices. Then,

$$
\begin{aligned}
& \mathrm{GA}(G)=9 n^{2}+\left(\frac{24 \sqrt{6}}{5}-15\right) n+12-\frac{24}{5} \sqrt{6} \\
& \operatorname{ABC}(G)=6 n^{2}+(6 \sqrt{2}-10) n+4-3 \sqrt{2}, \\
& \operatorname{AZI}(G)=\frac{729}{64}\left(9 n^{2}-15 n+6\right)+96 n-48
\end{aligned}
$$

Proof. Let $G$ be a cellular network. Then, one has

$$
\begin{aligned}
& \mathrm{GA}(G)=\sum_{i j \in E(G)} \frac{2 \sqrt{d_{i} \times d_{j}}}{d_{i}+d_{j}} \\
& =6 \times \frac{2 \sqrt{2 \times 2}}{2+2}+(12 n-12) \times \frac{2 \sqrt{2 \times 3}}{2+3} \\
& +\left(9 n^{2}-15 n+6\right) \times \frac{2 \sqrt{3 \times 3}}{3+3} \\
& =6 \times \frac{2 \times 2}{4}+(12 n-12) \times \frac{2 \sqrt{6}}{5} \\
& +\left(9 n^{2}-15 n+6\right) \times \frac{2 \times 3}{6} \\
& =9 n^{2}+\left(\frac{24 \sqrt{6}}{5}-15\right) n+12-\frac{24}{5} \sqrt{6}, \\
& \operatorname{ABC}(G)=\sum_{i j \in E(G)} \sqrt{\frac{d_{i}+d_{j}-2}{d_{i} \times d_{j}}} \\
& =6 \times \sqrt{\frac{2+2-2}{2 \times 2}}+(12 n-12) \times \sqrt{\frac{2+3-2}{2 \times 3}} \\
& +\left(9 n^{2}-15 n+6\right) \times \sqrt{\frac{3+3-2}{3 \times 3}} \\
& =3 \sqrt{2}+(6 n-6) \sqrt{2}+\left(6 n^{2}-10 n+4\right) \times 2 \\
& =6 n^{2}+(6 \sqrt{2}-10) n+4-3 \sqrt{2}, \\
& \operatorname{AZI}(G)=\sum_{i j \in E(G)}\left(\frac{d_{i} \times d_{j}}{d_{i}+d_{j}-2}\right)^{3} \\
& =6 \times\left(\frac{2 \times 2}{2+2-2}\right)^{3}+(12 n-12) \times\left(\frac{2 \times 3}{2+3-2}\right)^{3} \\
& +\left(9 n^{2}-15 n+6\right) \times\left(\frac{3 \times 3}{3+3-2}\right)^{3} \\
& =6 \times 2^{3}+(12 n-12) \times 2^{3}+\left(9 n^{2}-15 n+6\right) \times\left(\frac{9}{4}\right)^{3} \\
& =\frac{729}{64}\left(9 n^{2}-15 n+6\right)+96 n-48 .
\end{aligned}
$$

Next, our step is to study the generalized Sierpiński networks $s\left(K_{p}, m\right)$ when its subgraph is a complete graph. By consulting [32], the edges of the $s\left(K_{p}, m\right)$ can be divided into two sets of vertex division based on valencies, as $A_{1}$ and $A_{2}$. The set $A_{1}$ consists of $m(m-1)$ edges $i j$, where $d_{i}=m$ and $d_{j}=m+1$. The set $A_{2}$ consists of $\left(m^{t+1}-2 m^{2}+\right.$ $m) / 2$ edges $i j$, where $d_{i}=m+1$ and $d_{j}=m+1$. 
Theorem 6. Suppose $G$ is a Sierpiński networks and its subgraph is a complete graph. Then,

$$
\begin{aligned}
\Pi_{2}(G)= & m^{m(m-1)} \cdot(m+1)^{m^{t+1}-m^{2}}, \\
\Pi_{1}^{*}(G)= & (2 m+1)^{m(m-1)} \cdot(2 m+2)^{\left(m^{t+1}-2 m^{2}+m\right) / 2}, \\
F(G)= & m^{t+3}+2 m^{t+2}+m^{t+1}-3 m^{3}-m^{2}, \\
Z_{a, b}(G)= & \left(m^{2}-m\right) \cdot\left[m^{a}(m+1)^{b}+m^{b}(m+1)^{a}\right] \\
& +(m+1)^{a+b} \cdot\left(m^{t+1}-2 m^{2}+m\right) .
\end{aligned}
$$

Proof. Let $G$ be Sierpiński networks with the seed graph being acomplete graph. Then, one has

$$
\begin{aligned}
& \Pi_{2}(G)=\prod_{i j \in E(G)}\left(d_{i} \times d_{j}\right) \\
& =[m \cdot(m+1)]^{m(m-1)} \cdot\left[(m+1)^{2}\right]^{\left(m^{t+1}-2 m^{2}+m\right) / 2} \\
& =[m \cdot(m+1)]^{m(m-1)} \cdot(m+1)^{m^{t+1}-2 m^{2}+m} \\
& =m^{m(m-1)} \cdot(m+1)^{m^{t+1}-m^{2}}, \\
& \Pi_{1}^{*}(G)=\prod_{i j \in E(G)}\left(d_{i}+d_{j}\right) \\
& =[m+(m+1)]^{m(m-1)}+[(m+1)+(m+1)]^{\left(m^{t+1}-2 m^{2}+m\right) / 2} \\
& =(2 m+1)^{m(m-1)} \cdot(2 m+2)^{\left(m^{t+1}-2 m^{2}+m\right) / 2}, \\
& F(G)=\sum_{i j \in E(G)}\left(d_{i}^{2}+d_{j}^{2}\right) \\
& =\left[m^{2}+(m+1)^{2}\right] \cdot m(m-1)+\left[(m+1)^{2}+(m+1)^{2}\right] \frac{m^{t+1}-2 m^{2}+m}{2} \\
& =\left(2 m^{2}+2 m+1\right) \cdot\left(m^{2}-m\right)+\left(m^{2}+2 m+1\right) \cdot\left(m^{t+1}-2 m^{2}+m\right) \\
& =m^{t+3}+2 m^{t+2}+m^{t+1}-3 m^{3}-m^{2}, \\
& Z_{a, b}(G)=\sum_{i j \in E(G)}\left(d_{i}^{a} d_{j}^{b}+d_{i}^{b} d_{j}^{a}\right) \\
& =\left[m^{a}(m+1)^{b}+m^{b}(m+1)^{a}\right] \cdot m(m-1)+\frac{m^{t+1}-2 m^{2}+m}{2} \cdot\left[2(m+1)^{a}(m+1)^{b}\right] \\
& =\left(m^{2}-m\right) \cdot\left[m^{a}(m+1)^{b}+m^{b}(m+1)^{a}\right]+(m+1)^{a+b} \cdot\left(m^{t+1}-2 m^{2}+m\right) .
\end{aligned}
$$

Finally, we discuss the Sierpiński networks $s(p, m)$ when the seed graph is a $m$-regular graph without triangles. Similarly, the edges of the $s(p, m)$ can be divided into three sets of vertex division based on valencies, as $A_{1}, A_{2}$, and $A_{3}$. The set $A_{1}$ consists of $\left(\left(p^{t-1} m\right) / 2\right)(p-2 m)$ edges $i j$, where $d_{i}=m$ and $d_{j}=m$. The set $A_{2}$ consists of $\left(p^{t-1}+\left(\left(p^{t-1}-\right.\right.\right.$ $p) /(1-p))) m^{2}$ edges $i j$, where $d_{i}=m$ and $d_{j}=m+1$. The set $A_{3}$ consists of $(p m / 2)\left(\left(1-p^{t-1}\right) /(1-p)\right)+p m^{2}((1-$ $\left.p^{t-2}\right) /(1-p)$ ) edges $i j$, where $d_{i}=m+1$ and $d_{j}=m+1$.

Theorem 7. Suppose $G$ is a Sierpiński networks $s(p, m)$ and its subgraph is a m-regular graph without triangles. Then, 


$$
\begin{aligned}
\Pi_{2}(G)= & m^{p^{t-1} m(p-m)+\left(\left(p^{t-1}-p\right) /(1-p)\right) m^{2}} \cdot(m+1)^{p^{t-1} m^{2}+\left(\left(m^{2}\left(p-p^{t-1}\right)+p m\left(1-p^{t-1}\right)\right) /(1-p)\right)} \\
\Pi_{1}^{*}(G)= & 2^{\left(\left(p^{t-1} m\right) / 2\right)(p-2 m)+\left(\left(p m\left(1-p^{t-1}+2 m-2 m p^{t-2}\right)\right) /(2(1-p))\right)} \cdot m^{\left(\left(p^{t-1} m\right) / 2\right)(p-2 m)} \\
& \cdot(m+1)^{(p m / 2)\left(\left(1-p^{t-1}\right) /(1-p)\right)+p m^{2}\left(\left(1-p^{t-2}\right) /(1-p)\right)} \cdot(2 m+1)^{\left(p^{t-1}+\left(\left(p^{t-1}-p\right) /(1-p)\right)\right) m^{2}} \\
F(G)= & \left(p^{t-1}+\frac{p^{t-1}-p}{1-p}\right) m^{4}+p^{t-1}(p-2 m) m^{3}+\left[p^{t-1} m^{2}+\frac{m^{2}\left(p-p^{t-1}\right)+p m\left(1-p^{t-1}\right)}{1-p}\right](m+1)^{2}, \\
Z_{a, b}(G)= & p^{t-1}(p-2 m) \cdot m^{a+b+1}+\left(p^{t-1}+\frac{p^{t-1}-p}{1-p}\right) m^{2}\left[m^{a}(m+1)^{b}+m^{b}(m+1)^{a}\right] \\
& +\frac{p m\left(1-p^{t-1}+2 m-2 m p^{t-2}\right)}{1-p}(m+1)^{a+b} \cdot
\end{aligned}
$$

Proof. Let $G$ be Sierpiński networks and its subgraph be $m$-regular graph without triangles. Then, one has

$$
\begin{aligned}
& \Pi_{2}(G)=\prod_{i j \in E(G)}\left(d_{i} \times d_{j}\right) \\
& =(m \cdot m){ }^{\left(\left(p^{t-1} m\right) / 2\right)(p-2 m)} \cdot[m \cdot(m+1)]^{\left(p^{t-1}+\left(\left(p^{t-1}-p\right) /(1-p)\right)\right) m^{2}} \\
& \cdot[(m+1) \cdot(m+1)]^{(p m / 2)\left(\left(1-p^{t-1}\right) /(1-p)\right)+p m^{2}\left(\left(1-p^{t-2}\right) /(1-p)\right)} \\
& =m^{p^{t-1} m(p-2 m)} \cdot m^{\left(p^{t-1}+\left(\left(p^{t-1}-n\right) / 1-\right)\right) m^{2}} \cdot(m+1)\left(p^{t-1}+\left(\left(p^{t-1}-p\right) /(1-p)\right)\right) m^{2}+p m\left(\left(1-p^{t-1}\right) /(1-p)\right)+2 p m^{2}\left(\left(1-p^{t-2}\right) /(1-p)\right) \\
& =m^{p^{t-1} m(p-m)+\left(\left(p^{t-1}-p\right) /(1-p)\right) m^{2}} \cdot(m+1)^{p^{t-1} m^{2}+\left(\left(m^{2}\left(p-p^{t-1}\right)+p m\left(1-p^{t-1}\right)\right) /(1-p)\right),} \\
& \Pi_{1}^{*}(G)=\prod_{i j \in E(G)}\left(d_{i}+d_{j}\right) \\
& =(m+m)\left(\left(p^{t-1} m\right) / 2\right)(p-2 m) \cdot[m+(m+1)]^{\left(p^{t-1}+\left(\left(p^{t-1}-p\right) /(1-p)\right)\right) m^{2}} . \\
& \cdot[(m+1)+(m+1)]^{(p m / 2)\left(\left(1-p^{t-1}\right) /(1-p)\right)+p m^{2}\left(\left(1-p^{t-2}\right) /(1-n)\right)} \\
& =2^{\left(\left(p^{t-1} m\right) / 2\right)(p-2 m)+(p m / 2)\left(\left(1-p^{t-1}\right) /(1-p)\right)+p m^{2}\left(\left(1-p^{t-2}\right) /(1-p)\right)} \cdot m^{\left(\left(p^{t-1} m\right) / 2\right)(p-2 m)} \cdot(2 m+1)^{\left(p^{t-1}+\left(\left(p^{t-1}-p\right) /(1-p)\right)\right) m^{2}} \\
& \cdot(m+1)^{(p m / 2)\left(\left(1-p^{t-1}\right) /(1-p)\right)+p m^{2}\left(\left(1-p^{t-2}\right) /(1-p)\right)} \\
& =2^{\left(p^{t-1} m / 2\right)(p-2 m)+\left(\left(p m\left(1-p^{t-1}+2 m-2 m p^{t-2}\right)\right) /(2(1-p))\right)} \cdot m^{\left(p^{t-1} m / 2\right)(p-2 m)} \cdot(m+1)^{(p m / 2)\left(\left(1-p^{t-1}\right) /(1-p)\right)+p m^{2}\left(\left(1-p^{t-2}\right) /(1-p)\right)} \\
& \cdot(2 m+1)\left(p^{t-1}+\left(\left(p^{t-1}-p\right) /(1-p)\right)\right) m^{2},
\end{aligned}
$$




$$
\begin{aligned}
& F(G)=\sum_{i j \in E(G)}\left(d_{i}^{2}+d_{j}^{2}\right) \\
& =\left[m^{2}+m^{2}\right] \cdot \frac{p^{t-1} m}{2}(p-2 m)+\left(\left(p^{t-1}+\frac{p^{t-1}-p}{1-p}\right) m^{2}\right)\left[m^{2}+(m+1)^{2}\right] \\
& +\left[\frac{p m}{2}\left(\frac{1-p^{t-1}}{1-p}\right)+p m^{2}\left(\frac{1-p^{t-2}}{1-p}\right)\right] \cdot\left[(m+1)^{2}+(m+1)^{2}\right] \\
& =p^{t-1} m^{3}(p-2 m)+\left(p^{t-1}+\frac{p^{t-1}-p}{1-p}\right) m^{4}+\left(p^{t-1}+\frac{p^{t-1}-p}{1-p}\right) m^{2}(m+1)^{2} \\
& +\left[p m \frac{1-p^{t-1}}{1-p}+2 p m^{2} \frac{1-p^{t-2}}{1-p}\right] \cdot(m+1)^{2} \\
& =\left(p^{t-1}+\frac{p^{t-1}-p}{1-p}\right) m^{4}+p^{t-1}(p-2 m) m^{3}+\left[p^{t-1} m^{2}+\frac{m^{2}\left(p-p^{t-1}\right)+p m\left(1-p^{t-1}\right)}{1-p}\right](m+1)^{2}, \\
& Z_{a, b}(G)=\sum_{i j \in E(G)}\left(d_{i}^{a} d_{j}^{b}+d_{i}^{b} d_{j}^{a}\right) \\
& =\frac{p^{t-1} m}{2}(p-2 m) \cdot\left(m^{a} m^{b}+m^{b} m^{a}\right)+\left(p^{t-1}+\frac{p^{t-1}-p}{1-p}\right) m^{2} \cdot\left[m^{a}(m+1)^{b}+m^{b}(m+1)^{a}\right] \\
& +\frac{p m}{2}\left(\frac{1-p^{t-1}}{1-p}\right)+p m^{2}\left(\frac{1-p^{t-2}}{1-p}\right) \cdot\left(2(m+1)^{a+b}\right) \\
& =p^{t-1}(p-2 m) \cdot m^{a+b+1}+\left(p^{t-1}+\frac{p^{t-1}-p}{1-p}\right) m^{2}\left[m^{a}(m+1)^{b}+m^{b}(m+1)^{a}\right] \\
& +\frac{p m\left(1-p^{t-1}+2 m-2 m p^{t-2}\right)}{1-p}(m+1)^{a+b},
\end{aligned}
$$

as desired.

\section{Conclusion}

In this paper, we studied the $\mathrm{ABC}$ index of some chemical networks and obtained AZI index, GA index, the multiplicative version of ordinary first Zagreb index, the second multiplicative Zagreb indices, and the Zagreb index. By calculating the correlation index of several specific chemical networks, we can get the above indices formulas. This also provides potential help for scholars to study networks characteristics better. For further work, if the corresponding networks are replaced by other networks, we can also calculate and get the corresponding formulas.

\section{Data Availability}

The figures, tables, and other data used to support this study are included within the article.

\section{Conflicts of Interest}

The authors declare that there are no conflicts of interest regarding the publication of this paper.

\section{Acknowledgments}

This work was supported in part by Anhui Provincial Natural Science Foundation under Grant 2008085J01 and Natural Science Fund of Education Department of Anhui Province under Grant KJ2020A0478.

\section{References}

[1] H. Chen, R. Wu, and H. Deng, "The extremal values of some topological indices in bipartite graphs with a given matching number," Applied Mathematics and Computation, vol. 280, pp. 103-109, 2016.

[2] H. Deng, G. Huang, and X. Jiang, "A unified linearprogramming modeling of some topological indices," Journal of Combinatorial Optimization, vol. 30, no. 3, pp. 826-837, 2015.

[3] J.-B. Liu and X.-F. Pan, "Minimizing Kirchhoff index among graphs with a given vertex bipartiteness," Applied Mathematics and Computation, vol. 291, pp. 84-88, 2016.

[4] W. Cai, L. Huang, and S. Wang, "Class D power amplifier for medical application," Informatics Engineering, an International Journal, vol. 4, no. 2, pp. 09-15, 2016.

[5] W. Cai and L. L. Gouveia, "Modeling and simulation of maximum power point tracker in ptolemy," Journal of Clean Energy Technologies, vol. 1, pp. 6-9, 2014. 
[6] M. Dehmer, F. Emmert-Streib, and M. Grabner, "A computational approach to construct a multivariate complete graph invariant," Information Sciences, vol. 260, pp. 200-208, 2014.

[7] B. Furtula, I. Gutman, and M. Dehmer, "On structuresensitivity of degree-based topological indices," Applied Mathematics and Computation, vol. 219, no. 17, pp. 89738978, 2013.

[8] J. A. Bondy and U. S. R. Murty, Graph Theory with Applications, Macmillan, New York, NY, USA, 1976.

[9] E. Estrada, L. Torres, L. Rodríguez, and I. Gutman, "An atombond connectivity index: modelling the enthalpy of formation of alkanes," Indian Journal of Chemistry, vol. 37, pp. 849-855, 1998.

[10] D. Vukicevi and B. Furtula, "Topological index based on the ratios of geometrical and arithmetical means of end-vertex degrees of edges," Journal of Mathematical Chemistry, vol. 46, pp. 1369-1376, 2009.

[11] W. Gao, M. K. Siddiqui, M. Naeem, and M. Imran, "Computing multiple $A B C$ index and multiple GA index of some grid graphs," Open Physics, vol. 16, no. 1, pp. 588-598, 2018.

[12] B. Furtula, A. Graovac, and D. Vukičević, "Augmented zagreb index," Journal of Mathematical Chemistry, vol. 48, no. 2, pp. 370-380, 2010.

[13] M. Eliasi, A. Iranmanesh, and I. Gutman, "Multiplicative version of first zagreb index," MATCH Communications in Mathematical and in Computer Chemistry, vol. 68, pp. 217230, 2012.

[14] B. Zhou and N. Trinajstić, "On general sum-connectivity index," Journal of Mathematical Chemistry, vol. 47, no. 1, pp. 210-218, 2010.

[15] L. Berrocal, A. Olivieri, and J. Rada, "Extremal values of vertex-degree-based topological indices over hexagonal systems with fixed number of vertices," Applied Mathematics and Computation, vol. 243, pp. 176-183, 2014.

[16] R. Kazemi, "Note on the multiplicative Zagreb indices," Discrete Applied Mathematics, vol. 198, pp. 147-154, 2016.

[17] C. Wang, S. Wang, and B. Wei, "Cacti with extremal PI index," Transition Comb.vol. 5, pp. 1-8, 2016.

[18] S. Wang, M. Farahani, A. Baig, and W. Sajja, "The sadhana polynomial and the sadhana index of polycyclic aromatic hydrocarbons PAHk," Journal of Chemical and Pharmaceutical Research.vol. 8, pp. 526-531, 2016.

[19] R. M. Tache, "On degree-based topological indices for bicyclic graphs," MATCH Communications in Mathematical and in Computer, vol. 76, pp. 99-116, 2016.

[20] I. Gutman, "Multiplicative Zagreb indices of trees," Bulletin of Society of Mathematicians Banja Luka, vol. 18, pp. 17-23, 2011.

[21] M. Azari and A. Iranmanesh, "Generalized Zagreb index of graphs,” Studia Univ Babes-Bolyai, vol. 56, no. 3, pp. 59-70, 2011.

[22] I. Gutman and N. N. Trinajstić, "Graph theory and molecular orbitals. Total $\varphi$-electron energy of alternant hydrocarbons," Chemical Physics Letters, vol. 17, no. 4, pp. 535-538, 1972.

[23] P. Sarkar, N. De, and A. Pal, "F-index of graphs based on new operations related to the join of Graphs," 2017, https://arxiv. org/pdf/1709.06301.pdf.

[24] N. De, S. M. A. Nayeem, and A. Pal, "F-index of some graph operations," Discrete Math Algorithm Appl, vol. 8, no. 2, pp. 1-17, 2016.

[25] N. De and S. M. A. Nayeem, "Computing the F-index of nanostar dendrimers," Pacific Science Review A: Natural Science and Engineering, vol. 18, no. 1, pp. 14-21, 2016.
[26] N. De, "F-index and coindex of some derived graphs," Journal of the International Mathematical Virtual Institute, vol. 8, pp. 81-88, 2018.

[27] S. Hayat and M. Imran, "Computation of topological indices of certain networks," Applied Mathematics and Computation, vol. 240, pp. 213-228, 2014.

[28] Y. Yang and H. Zhang, "Kirchhoffindex of linear hexagonal chains," International Journal of Quantum Chemistry, vol. 503-512, 2008.

[29] J. Huang, S. Li, and L. Sun, “The normalized Laplacians, degree-kirchhoff index and the spanning trees of linear hexagonal chains," Discrete Applied Mathematics, vol. 207, pp. 67-79, 2016.

[30] Y. Pan and J. Li, "Kirchhoff index, multiplicative degreeKirchhoff index and spanning trees of the linear crossed hexagonal chains," International Journal of Quantum Chemistry, vol. 118, Article ID e25787, , 2018.

[31] X. Ma and H. Bian, "The normalized Laplacians, degreeKirchhoff index and the spanning trees of hexagonal Möbius graphs," Applied Mathematics and Computation, vol. 355, pp. 33-46, 2019.

[32] J.-B. Liu, J. Zhao, H. He, and Z. Shao, "Valency-based topological descriptors and structural property of the generalized Sierpiński networks," Journal of Statistical Physics, vol. 177, no. 6, pp. 1131-1147, 2019. 